\title{
$k$-distant crossings and nestings of matchings and partitions
}

\author{
Dan Drake ${ }^{1 \dagger}$ and Jang Soo Kim ${ }^{1}$ \\ ${ }^{1}$ Department of Mathematical Sciences \\ Korea Advanced Institute of Science and Technology \\ Daejeon, Korea
}

\begin{abstract}
We define and consider $k$-distant crossings and nestings for matchings and set partitions, which are a variation of crossings and nestings in which the distance between vertices is important. By modifying an involution of Kasraoui and Zeng (Electronic J. Combinatorics 2006, research paper 33), we show that the joint distribution of $k$-distant crossings and nestings is symmetric. We also study the numbers of $k$-distant noncrossing matchings and partitions for small $k$, which are counted by well-known sequences, as well as the orthogonal polynomials related to $k$-distant noncrossing matchings and partitions. We extend Chen et al.'s $r$-crossings and enhanced $r$-crossings.

Résumé. Nous définissons les notions de croisements et imbrications $k$-distants sur les appariements et les partitions d'ensemble, qui sont une variation sur les notions usuelles prenant en compte la distance entre les sommets. En modifiant une involution de Kasraoui et Zeng (Electronic J. Combinatorics 2006, research paper 33), nous montrons que la distribution jointe des croisements et imbrications $k$-distants est symétrique. Nous étudions le nombre d'involutions et de partitions sans croisement $k$-distant pour de petites valeurs de $k$, qui sont des suites d'entiers bien connues, ainsi que les polynômes orthogonaux qui leur sont reliés. Nous étendons les notions de $r$-croisements et $r$-croisements amliorés dues à Chen et al.
\end{abstract}

Keywords: crossings, nestings, set partitions, matchings

\section{Introduction}

A (set) partition of $[n]=\{1,2, \ldots, n\}$ is a set of disjoint subsets of $[n]$ whose union is $[n]$. Each element of a partition is called a block. We will write a partition as a sequence of blocks, for instance, $\{1,4,8\}\{2,5,9\}\{3\}\{6,7\}$. Let $\Pi_{n}$ denote the set of partitions of $[n]$.

Let $\pi$ be a partition of $[n]$. A vertex of $\pi$ is an integer $i \in[n]$. An edge of $\pi$ is a pair $(i, j)$ of vertices satisfying either (1) $i<j$, and $i$ and $j$ are in the same block with no vertex between them in that block, or (2) $i=j$ and the block containing $i$ has no other vertex. Thus when we arrange vertices of $\pi=\{1,5\}\{2,4,9\}\{3\}\{6,12\}\{7,10,11\}\{8\}$, in a line in increasing order and draw edges we get Figure 1 .

\footnotetext{
${ }^{\dagger}$ The first author was supported by the second stage of the Brain Korea 21 Project, The Development Project of Human Resources in Mathematics, KAIST in 2008.
} 


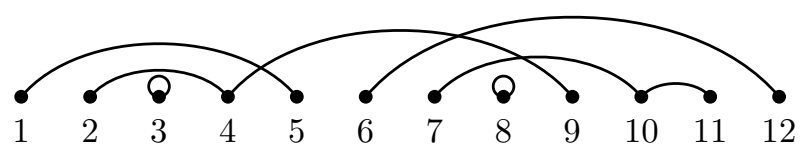

Fig. 1: Diagram for $\{1,5\}\{2,4,9\}\{3\}\{6,12\}\{7,10,11\}\{8\}$.

A vertex $v$ of $\pi$ is called an opener (resp. closer) if $v$ is the smallest (resp. largest) element of a block consisting of at least two integers. A vertex $v$ is called a singleton if $v$ itself makes a block. A vertex $v$ is called a transient if there are two edges connected to $v$. Let $\mathcal{O}(\pi)$ (resp. $\mathcal{C}(\pi), \mathcal{S}(\pi), \mathcal{T}(\pi)$ ) be the set of openers (resp. closers, singletons, transients) of $\pi$. Let type $(\pi)=(\mathcal{O}(\pi), \mathcal{C}(\pi), \mathcal{S}(\pi), \mathcal{T}(\pi))$ and type $(\pi)=(\mathcal{O}(\pi), \mathcal{C}(\pi), \mathcal{S}(\pi) \cup \mathcal{T}(\pi))$. For the partition in Figure 1, the type of $\pi$ is type $(\pi)=$ $(\{1,2,6,7\},\{5,9,11,12\},\{3,8\},\{4,10\})$.

A (complete) matching is a partition without singletons or transients; this is the same thing as a partition in which all blocks have size 2 .

Now we can define the main object of our study.

Definition. Let $k$ be a nonnegative integer. A $k$-distant crossing of $\pi$ is a pair of edges $\left(i_{1}, j_{1}\right)$ and $\left(i_{2}, j_{2}\right)$ of $\pi$ satisfying $i_{1}<i_{2} \leq j_{1}<j_{2}$ and $j_{1}-i_{2} \geq k$. A $k$-distant nesting of $\pi$ is a set of two edges $\left(i_{1}, j_{1}\right)$ and $\left(i_{2}, j_{2}\right)$ of $\pi$ satisfying $i_{1}<i_{2} \leq j_{2}<j_{1}$ and $j_{2}-i_{2} \geq k$.

Let $\operatorname{dcr}_{k}(\pi)$ (resp. $\operatorname{dne}_{k}(\pi)$ ) denote the number of $k$-distant crossings ( $k$-distant nestings) in $\pi$. Thus $\operatorname{dcr}_{1}(\pi)$ is the number of usual crossings of $\pi$.

For example, in the partition in Figure 1, the edges $(4,9)$ and $(6,12)$ form a 3 -distant crossing (as well as an $i$-distant crossing for $i=0,1,2)$, the edges $(1,5)$ and $(2,4)$ form a 2 -distant nesting, the edges $(2,4)$ and $(4,9)$ form a 0 -distant crossing, and the edges $(7,10)$ and $(8,8)$ form a 0 -distant nesting. That partition has $\operatorname{dcr}_{0}(\pi)=5, \operatorname{dcr}_{2}(\pi)=2$, and $\operatorname{dne}_{2}(\pi)=2$.

Kasraoui and Zeng [5] found an involution $\varphi: \Pi_{n} \rightarrow \Pi_{n}$ such that type $(\varphi(\pi))=\operatorname{type}(\pi)$ and $\operatorname{dcr}_{1}(\varphi(\pi))=$ dne $_{1}(\pi)$, dne ${ }_{1}(\varphi(\pi))=\operatorname{dcr}_{1}(\pi)$. Modifying this involution, for $k \geq 0$, we find an involution $\varphi_{k}: \Pi_{n} \rightarrow \Pi_{n}$ such that $\operatorname{dcr}_{k}\left(\varphi_{k}(\pi)\right)=\operatorname{dne}_{k}(\pi), \operatorname{dne}_{k}\left(\varphi_{k}(\pi)\right)=\operatorname{dcr}_{k}(\pi)$ and type $\left(\varphi_{k}(\pi)\right)=$ $\operatorname{type}(\pi)$ if $k \geq 1$; $\operatorname{type}^{\prime}\left(\varphi_{k}(\pi)\right)=\operatorname{type}^{\prime}(\pi)$ if $k=0$.

Noncrossing partitions and matchings are interesting and pervasive objects that arise frequently in diverse areas of mathematics; see [10] and [11] and the references therein for an introduction to noncrossing partitions. A partition $\pi$ is called $k$-distant noncrossing if $\pi$ has no $k$-distant crossing. Let $N C M_{k}(n)$ denote the number of $k$-distant noncrossing matchings of $[n]$. Let $N C P_{k}(n)$ denote the number of $k$-distant noncrossing partitions of $[n]$.

Table 1 and Table 2 show $N C M_{k}(n)$ and $N C P_{k}(n)$ for small values of $n$ and $k$. We use $k=\infty$ to indicate that $i$-distant crossing is allowed for any positive integer $i$, so that $N C M_{\infty}(n)$ and $N C P_{\infty}(n)$ equal the total number of matchings of $[2 n]$ and partitions of $[n]$, respectively. A matching or partition cannot have a $k$-distant crossing for $k>n-3$, so for fixed $n, N C M_{k}(n)$ and $N C P_{k}(n)$ will "converge" to the number of matchings and number of partitions, respectively; for readability we omit those numbers in the tables. The $n=0$ column is all 1's for both tables, of course.

It is well known that noncrossing matchings of $[2 n]$ and noncrossing partitions of $[n]$ are counted by the Catalan number $C_{n}$. Thus $N C M_{0}(2 n)=N C M_{1}(2 n)=N C P_{1}(n)=C_{n}$. We will show that $N C M_{2}(2 n)=s_{n}$ and $N C P_{0}(n)=M_{n}$, where $s_{n}$ and $M_{n}$ are the little Schröder numbers (A001003 in [12]) and the Motzkin numbers (A001006 in [12]) respectively. We will also find the generating functions 


\begin{tabular}{|c|c|c|c|c|c|c|c|c|c|c|}
\hline$k \backslash n$ & 2 & 4 & 6 & 8 & 10 & 12 & 14 & 16 & 18 & 20 \\
\hline 1 & 1 & 2 & 5 & 14 & 42 & 132 & $\overline{429}$ & 1430 & 4862 & 16796 \\
\hline 2 & & 3 & 11 & 45 & 197 & 903 & 4279 & 20793 & 103049 & 518859 \\
\hline 3 & & & 14 & 71 & 387 & 2210 & 13053 & 79081 & 488728 & 3069007 \\
\hline 4 & & & 15 & 91 & 581 & 3906 & 27189 & 194240 & 1416168 & 10494328 \\
\hline 5 & & & & 102 & 753 & 5752 & 45636 & 372360 & 3101523 & 26266917 \\
\hline 6 & & & & 105 & 873 & 7541 & 66690 & 607128 & 5657520 & 53631564 \\
\hline 7 & & & & & 930 & 8985 & 88450 & 885394 & 9067611 & 94719138 \\
\hline 8 & & & & & 945 & 9885 & 107847 & 1187376 & 13233511 & 150234570 \\
\hline 9 & & & & & & 10290 & 122115 & 1476948 & 17933348 & 219754737 \\
\hline 10 & & & & & & 10395 & 130515 & 1715475 & 22701570 & 300724081 \\
\hline 11 & & & & & & & 134190 & 1881495 & 26969370 & 386669322 \\
\hline 12 & & & & & & & 135135 & 1975995 & 30306045 & 468680940 \\
\hline 13 & & & & & & & & 2016630 & 32546745 & 538581120 \\
\hline 14 & & & & & & & & 2027025 & 33794145 & 591287445 \\
\hline 15 & & & & & & & & & 34324290 & 625810185 \\
\hline 16 & & & & & & & & & 34459425 & 652702050 \\
\hline 17 & & & & & & & & & & 644729085 \\
\hline 18 & & & & & & & & & & 654729075 \\
\hline$\infty$ & 1 & 3 & 15 & 105 & 945 & 10395 & 135135 & 2027025 & 34459425 & 654729075 \\
\hline
\end{tabular}

Tab. 1: $k$-distant noncrossing matchings. The $k=0$ row is omitted because, as matchings have no transient vertices, the $k=0$ row is the same as $k=1$ row; both, of course, are counted by the Catalan numbers (A000108). The $k=2$ row is the little Schröder numbers (A001003).

\begin{tabular}{r|rrrrrrrrrrrr}
$k \backslash n$ & 1 & 2 & 3 & 4 & 5 & 6 & 7 & 8 & 9 & 10 & 11 & 12 \\
\hline 0 & 1 & 2 & 4 & 9 & 21 & 51 & 127 & 323 & 835 & 2188 & 5798 & 15511 \\
1 & & & 5 & 14 & 42 & 132 & 429 & 1430 & 4862 & 16796 & 58786 & 208012 \\
2 & & & & 15 & 51 & 188 & 731 & 2950 & 12235 & 51822 & 223191 & 974427 \\
3 & & & & & 52 & 201 & 841 & 3726 & 17213 & 82047 & 400600 & 1993377 \\
4 & & & & & & 203 & 872 & 4037 & 19796 & 101437 & 537691 & 2926663 \\
5 & & & & & & & 877 & 4125 & 20802 & 110950 & 618777 & 3575688 \\
6 & & & & & & & & 4140 & 21095 & 114663 & 657698 & 3943294 \\
7 & & & & & & & & & 21147 & 115772 & 673019 & 4118232 \\
8 & & & & & & & & & & 115975 & 677693 & 4187838 \\
9 & & & & & & & & & & & 678570 & 4209457 \\
10 & & & & & & & & & & & & 4213597 \\
\hline$\infty$ & 1 & 2 & 5 & 15 & 52 & 203 & 877 & 4140 & 21147 & 115975 & 678570 & 4213597
\end{tabular}

Tab. 2: $k$-distant noncrossing partitions. The $k=0,1$, and 2 rows are counted by Motzkin numbers (A001006), the Catalan numbers, and A007317, respectively. 


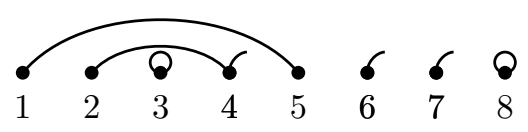

Fig. 2: The 8-th trace $T_{8}(\pi)$ of $\pi$ in Figure 1 . The vacant vertices are 4,6 and 7 .

for $\mathrm{NCP}_{2}(n)$ and $\mathrm{NCM}_{3}(2 n)$.

Throughout this paper we will frequently refer to sequences in the Online Encyclopedia of Integer Sequences [12] using their "A number"; we will usually omit the citation to [12] and consider it understood that things like "A000108" are a reference to the corresponding sequence in the OEIS.

The rest of this paper is organized as follows. In section 2, we modify Kasraoui and Zeng's involution to prove the joint distribution of $k$-distant crossings and nestings is symmetric. In section 3 , we review a bijection between partitions and Charlier diagrams. In section 4 and section 5, we study the number of $k$-distant noncrossing matchings and partitions, and, in section 6 , we consider the orthogonal polynomials related to these numbers. In section 7, we extend $r$-crossings and enhanced $r$-crossings of Chen et al. [1].

\section{Modification of the involution of Kasraoui and Zeng}

Kasraoui and Zeng [5] found an involution $\varphi: \Pi_{n} \rightarrow \Pi_{n}$ such that $\operatorname{dcr}_{1}(\varphi(\pi))=\operatorname{dne}_{1}(\pi)$, dne $1(\varphi(\pi))=$ $\operatorname{dcr}_{1}(\pi)$ and type $(\varphi(\pi))=\operatorname{type}(\pi)$. In this section, for fixed $k \geq 0$, we find an involution $\varphi_{k}: \Pi_{n} \rightarrow \Pi_{n}$ such that $\operatorname{dcr}_{k}\left(\varphi_{k}(\pi)\right)=\operatorname{dne}_{k}(\pi)$ and $\operatorname{dne}_{k}\left(\varphi_{k}(\pi)\right)=\operatorname{dcr}_{k}(\pi)$.

We will follow Kasraoui and Zeng's notations. We will identify a partition $\pi$ to its diagram as shown in Figure 1.

The $i$-th trace $T_{i}(\pi)$ of $\pi$ is the diagram obtained from $\pi$ by removing vertices greater than $i$. If a vertex $v \leq i$ is connected to $u>i$ in $\pi$ then make a half edge from $v$ in $T_{i}(\pi)$. Each vertex with a half edge is called vacant vertex. For an example, see Figure 2.

Let $k$ be a fixed nonnegative integer. We define $\varphi_{k}: \Pi_{n} \rightarrow \Pi_{n}$ as follows.

1. Set $T_{0}^{(k)}=\emptyset$.

2. For $1 \leq i \leq n, T_{i}^{(k)}$ is obtained as follows.

(a) Let $T_{i}^{(k)}\left(\operatorname{resp} . T_{i}^{\prime}(\pi)\right)$ be $T_{i-1}^{(k)}\left(\right.$ resp. $\left.T_{i-1}(\pi)\right)$ with new vertex $i$.

(b) If $i \in \mathcal{O}(\pi) \cup \mathcal{S}(\pi) \cup \mathcal{T}(\pi)$, then make a half edge from $i$ both in $T_{i}^{(k)}$ and $T_{i}^{\prime}(\pi)$.

(c) If $i \in \mathcal{C}(\pi) \cup \mathcal{S}(\pi) \cup \mathcal{T}(\pi)$, let $j$ be the vertex connected to $i$ in $\pi$.

i. If $i-j<k$, then $j$ must be a vacant vertex in $T_{i}^{(k)}$. Remove the half edge from $j$ and add an edge $(i, j)$ in $T_{i}^{(k)}$.

ii. If $i-j \geq k$, then let $U$ (resp. $V$ ) be the set of all vacant vertices $v$ in $T_{i}^{(k)}\left(\right.$ resp. $T_{i}^{\prime}(\pi)$ ) such that $i-v \geq k$. Let $\gamma_{i}^{(k)}(\pi)$ denote the integer $r$ such that $j$ is the $r$-th largest element of $V$. Let $j^{\prime}$ be the $\gamma_{i}^{(k)}(\pi)$-th smallest element of $U$. Remove the half edge from $j^{\prime}$ and add an edge $\left(j^{\prime}, i\right)$ in $T_{i}^{(k)}$. 


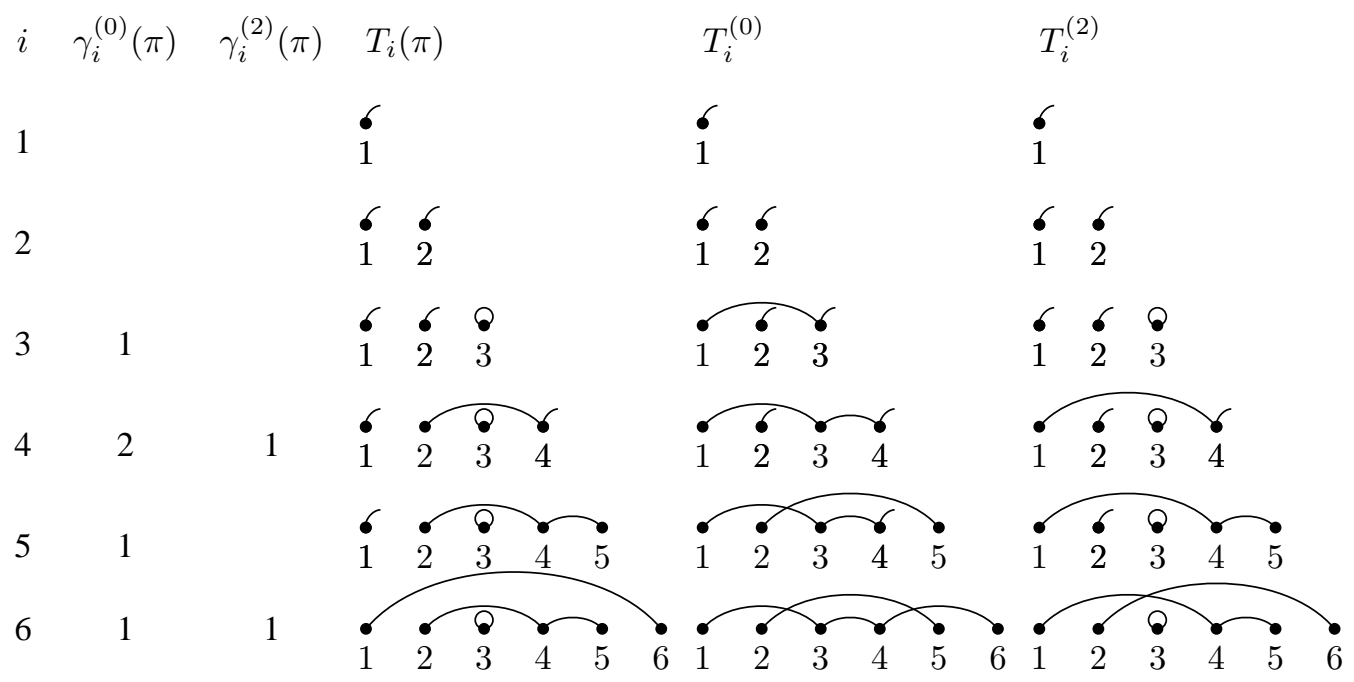

Fig. 3: Construction of $\varphi_{0}(\pi)=T_{6}^{(0)}$ and $\varphi_{2}(\pi)=T_{6}^{(2)}$ for $\pi=\{1,6\}\{2,4,5\}\{3\}$.

3. Set $\varphi_{k}(\pi)=T_{n}^{(k)}$.

For example, see Figure 3. Using the same argument as in [5], we can prove that $\varphi_{k}$ is an involution and satisfies $\operatorname{dcr}_{k}\left(\varphi_{k}(\pi)\right)=\operatorname{dne}_{k}(\pi), \operatorname{dne}_{k}\left(\varphi_{k}(\pi)\right)=\operatorname{dcr}_{k}(\pi)$, type $\left(\varphi_{k}(\pi)\right)=\operatorname{type}(\pi)$ if $k \geq 1$; $\operatorname{type}^{\prime}\left(\varphi_{k}(\pi)\right)=\operatorname{type}^{\prime}(\pi)$ if $k=0$. Thus we have the following.

Theorem 2.1. Let $k$ be a nonnegative integer. Then

$$
\sum_{\pi \in \Pi_{n}} x^{\mathrm{dcr}_{k}(\pi)} y^{\mathrm{dne}_{k}(\pi)}=\sum_{\pi \in \Pi_{n}} x^{\mathrm{dne}_{k}(\pi)} y^{\mathrm{dcr}_{k}(\pi)} .
$$

\section{Motzkin paths and Charlier diagrams}

In this section, we recall a bijection between partitions and Charlier diagrams $[4,5]$.

A step is a pair $(p, q)$ of points $p, q \in \mathbb{Z} \times \mathbb{Z}$. The height of a step $(p, q)$ is the second component of $p$, i.e, if $p=(a, b)$ then the height of the step $(p, q)$ is $b$. A step $(p, q)$ is called an $u p$ (resp. down, horizontal) step if the component-wise difference $q-p$ is $(1,1)$ (resp. $(1,-1),(1,0)$ ). A path of length $n$ is a sequence $\left(p_{0}, p_{1}, p_{2}, \ldots, p_{n}\right)$ of $n+1$ points in $\mathbb{Z} \times \mathbb{Z}$. The $i$-th step of a path $\left(p_{0}, p_{1}, p_{2}, \ldots, p_{n}\right)$ is $\left(p_{i-1}, p_{i}\right)$. A nonnegative path of length $n$ is a path from $(0,0)$ to $(n, 0)$, which never goes below the $x$-axis. A Motzkin path of length $n$ is a nonnegative path of length $n$ consisting of up steps, down steps and horizontal steps. A Charlier diagram of length $n$ is a pair $(M, e)$ where $M=\left(p_{0}, p_{1}, \ldots, p_{n}\right)$ is a Motzkin path of length $n$ and $e=\left(e_{1}, e_{2}, \ldots, e_{n}\right)$ is a sequence of integers such that

1. if the $i$-th step is an up step then $e_{i}=0$,

2. if the $i$-th step is a down step of height $h$ then $1 \leq e_{i} \leq h$,

3. if the $i$-th step is a horizontal step of height $h$ then $0 \leq e_{i} \leq h$. 


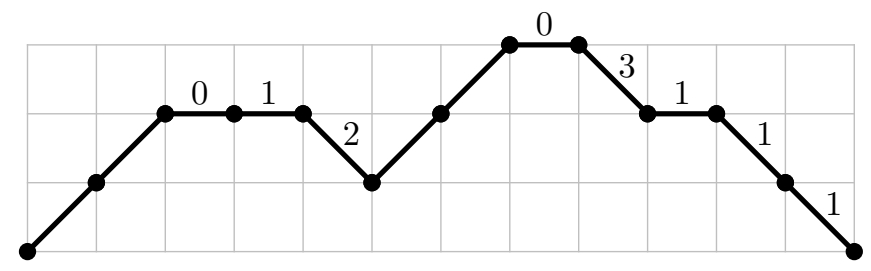

Fig. 4: The Charlier diagram for the partition of Figure 1. The label $e_{i}$ is written above the horizontal and down steps.

We will identify a Charlier diagram $(M, e)$ with the sequence $\left(s_{1}, s_{2}, \ldots, s_{n}\right)$ of labeled letters in $\left\{U, D_{1}, D_{2}, \ldots, H_{0}, H_{1}, H_{2}, \ldots\right\}$ such that $s_{i}=U$ (resp. $s_{i}=D_{e_{i}}, s_{i}=H_{e_{i}}$ ) if the $i$-th step of $M$ is an up (resp. down, horizontal) step.

Let $\pi$ be a partition of $[n]$. Recall that in the previous section, if $i$ is a closer or transient, then $\gamma_{i}^{(1)}(\pi)$ is the integer $r$ such that $i$ is connected to the $r$-th largest integer in $T_{i-1}^{(1)}(\pi)$.

The corresponding Charlier diagram $\operatorname{Ch}(\pi)=\left(s_{1}, s_{2}, \ldots, s_{n}\right)$ is defined as follows:

1. if $i$ is an opener in $\pi$ then $s_{i}=U$,

2. if $i$ is a closer in $\pi$ and $\gamma_{i}^{(1)}(\pi)=r$ then $s_{i}=D_{r}$,

3. if $i$ is a singleton in $\pi$ then $s_{i}=H_{0}$,

4. if $i$ is a transient in $\pi$ and $\gamma_{i}^{(1)}(\pi)=r$ then $s_{i}=H_{r}$.

For example, see Figure 4.

It is easy to see that if there is a step $D_{\ell}$ or $H_{\ell}$ with $\ell \geq 2$ in $\operatorname{Ch}(\pi)$, than $\pi$ has an $(\ell-1)$-distant crossing.

\section{$4 k$-distant noncrossing matchings}

In this section we will find the number of $k$-distant noncrossing matchings for $k=0,1,2$ and 3 . Note that since there is no matching of $[2 n+1]$ we have $N C M_{k}(2 n+1)=0$ for all $n$ and $k$. Thus we will only consider $N C M_{k}(2 n)$.

\subsection{0- and 1-distant noncrossing matchings}

Since matchings have no transient vertices, being 0-distant crossing is equivalent to being 1-distant crossing.

We can easily see that a matching $\pi$ is 1-distant noncrossing if and only if $\mathrm{Ch}(\pi)$ consists of $U$ and $D_{1}$. Thus a 1-distant noncrossing matching corresponds to a Dyck path.

Theorem 4.1. We have

$$
N C M_{0}(2 n)=N C M_{1}(2 n)=C_{n}=\frac{1}{n+1}\left(\begin{array}{c}
2 n \\
n
\end{array}\right)
$$




\subsection{2-distant noncrossing matchings}

Let $\pi$ be a 2-distant noncrossing matching. Then $\operatorname{Ch}(\pi)$ consists of $U, D_{1}$, and $D_{2}$. By definition of $\mathrm{Ch}(\pi), D_{2}$ is of height at least 2. Moreover, since $\pi$ has no 2-distant crossing, $D_{2}$ must immediately follow $U$. Thus we can consider $\operatorname{Ch}(\pi)$ as a nonnegative path consisting of the three steps $U=(1,1)$, $D_{1}=(1,-1)$ and $U D_{2}=(2,0)$ such that $U D_{2}$ never touches the $x$-axis. This is exactly the definition of a little Schröder path, see [13]. Thus we get the following theorem.

Theorem 4.2. We have

$$
\mathrm{NCM}_{2}(2 n)=s_{n},
$$

where $s_{n}$ is the little Schröder number (A001003).

\subsection{3-distant noncrossing matchings}

Let $\pi$ be a 3-distant noncrossing matching. One can check that $\operatorname{Ch}(\pi)$ consists of $U, D_{1}, D_{2}$, and $D_{3}$ satisfying the following.

1. $D_{\ell}$ is of height at least $\ell$ for $\ell=1,2,3$.

2. $D_{3}$ can only occur after two consecutive $U$, and

3. $D_{2}$ can only occur after $U$ or after either $D_{2}$ or $D_{3}$ which follows $U$.

Thus we can consider $\mathrm{Ch}(\pi)$ as a nonnegative path consisting of the 6 steps $U, D_{1}, U D_{2}, U U D_{3}$, $U D_{2} D_{2}, U U D_{3} D_{2}$ such that the last four steps must be above the line $y=1$. Let $g(n)$ be the number of nonnegative paths of length $n$ consisting of $U, D_{1}, U D_{2}, U U D_{3}, U D_{2} D_{2}$, and $U U D_{3} D_{2}$. Let $F(x)=$ $\sum_{n \geq 0} N C M_{3}(2 n) x^{n}$ and $G(x)=\sum_{n \geq 0} g(2 n) x^{n}$.

Decomposing nonnegative paths, we get that $G(x)=1+\left(x+x^{2}\right) G(x)+\left(x^{\frac{1}{2}}+x^{\frac{3}{2}}\right)^{2} G(x)^{2}$ and $F(x)=1+x G(x) F(x)$. Thus we get

$$
G(x)=\frac{1-x-x^{2}-\sqrt{1-6 x-9 x^{2}-2 x^{3}+x^{4}}}{2 x(x+1)^{2}} .
$$

Now we get the generating function for $\mathrm{NCM}_{3}(2 n)$.

Theorem 4.3. We have

$$
\begin{aligned}
\sum_{n \geq 0} N C M_{3}(2 n) x^{n} & =\frac{2(x+1)^{2}}{1+5 x+3 x^{2}+\sqrt{1-6 x-9 x^{2}-2 x^{3}+x^{4}}} \\
& =1+x+3 x^{2}+14 x^{3}+71 x^{4}+387 x^{5}+2210 x^{6}+13053 x^{7}+\cdots .
\end{aligned}
$$

\section{$5 k$-distant noncrossing partitions}

\subsection{0-distant noncrossing partitions}

Let $\pi$ be a 0 -distant noncrossing partition. Then $\operatorname{Ch}(\pi)$ consists of $U, D_{1}, H_{0}$. Thus $\operatorname{Ch}(\pi)$ is a Motzkin path.

Theorem 5.1. The number of 0 -distant noncrossing partitions of $[n]$ is equal to the number of Motzkin paths of length $[n]$ (A001006). 


\subsection{1-distant noncrossing partitions}

Let $\pi$ be a 1-distant noncrossing partition. Then $\pi$ is a usual noncrossing partition. It is well known that the number of noncrossing partitions of $[n]$ is the Catalan number $C_{n}$.

Theorem 5.2. We have

$$
N C P_{1}(n)=C_{n}
$$

\subsection{2-distant noncrossing partitions}

Let $\pi$ be a 2-distant noncrossing partition. Then $\operatorname{Ch}(\pi)$ consists of $U, D_{1}, D_{2}, H_{0}, H_{1}$, and $H_{2}$ and satisfies

1. $D_{\ell}$ and $H_{\ell}$ are of height at least $\ell$,

2. $H_{2}$ and $D_{2}$ can only occur after $U, H_{1}$, or $H_{2}$.

Thus we can consider $\mathrm{Ch}(\pi)$ as a nonnegative path with the following steps:

$$
U H_{2}^{k}, U H_{2}^{k} D_{2}, H_{1} H_{2}^{k}, H_{1} H_{2}^{k} D_{2}, H_{0} \text {, and } D_{1},
$$

where $k$ is a nonnegative integer and $H_{2}^{k}$ means $k$ consecutive $H_{2}$ steps.

Let $a(n)$ (resp. $b(n)$ ) denote the number of nonnegative paths of length $n$ consisting of the above steps such that $D_{\ell}$ and $H_{\ell}$ is of height at least $\ell-2$ (resp. at least $\ell-1$ ). In fact, the height condition is unnecessary for $a(n)$ since every step is of height at least 0 . Let $F(x)=\sum_{n \geq 0} N C P_{2}(n) x^{n}, A(x)=$ $\sum_{n \geq 0} a(n) x^{n}$, and $B(x)=\sum_{n \geq 0} b(n) x^{n}$.

Note that the steps which increase the $y$-coordinate by 1 are

$$
U H_{2}^{k}, \quad k \geq 0,
$$

the steps which do not change the $y$-coordinate are

$$
H_{0}, H_{1} H_{2}^{k}, U H_{2}^{k} D_{2}, \quad k \geq 0,
$$

and the steps which decrease the $y$-coordinate by 1 are

$$
D_{1}, H_{1} H_{2}^{k} D_{2}, \quad k \geq 0 .
$$

Thus, by decomposing nonnegative paths, we get

$$
\begin{aligned}
& A(x)=1+\left(x+\frac{x}{1-x}+\frac{x^{2}}{1-x}\right) A(x)+\frac{x}{1-x} \cdot\left(x+\frac{x^{2}}{1-x}\right) A(x)^{2}, \\
& B(x)=1+\left(2 x+\frac{x^{2}}{1-x}\right) B(x)+\frac{x}{1-x} \cdot\left(x+\frac{x^{2}}{1-x}\right) A(x) B(x), \\
& F(x)=1+x F(x)+x^{2} B(x) F(x) .
\end{aligned}
$$

Solving these equations, we get the following theorem. 
Theorem 5.3. We have

$$
\begin{aligned}
\sum_{n \geq 0} N C P_{2}(n) x^{n} & =\frac{3-3 x-\sqrt{1-6 x+5 x^{2}}}{2(1-x)}=\frac{3}{2}-\frac{1}{2} \sqrt{\frac{1-5 x}{1-x}} \\
& =1+x+2 x^{2}+5 x^{3}+15 x^{4}+51 x^{5}+188 x^{6}+731 x^{7}+2950 x^{8}+\cdots .
\end{aligned}
$$

This sequence is A007317. Mansour and Severini [9] proved that the generating function for the number of 12312-avoiding partitions is equal to that in 5.3. Thus the number of 2-distant noncrossing partitions of $[n]$ is equal to the number of 12312 -avoiding partitions of $[n]$. Yan [16] found a bijection from 12312avoiding partitions of $[n]$ to UH-free Schröder paths of length $2 n-2$. Composing several bijections including Yan's bijection, Kim [7] found a bijection between 2-distant noncrossing partitions and 12312avoiding partitions.

\section{Orthogonal polynomials}

Given a sequence $\left\{\mu_{n}\right\}_{n \geq 0}$, one may try to define a sequence of polynomials $\left\{P_{n}(x)\right\}_{n \geq 0}$ that are orthogonal with respect to $\left\{\mu_{n}\right\}$; that is, if we define a measure with $\mu_{n}=\int x^{n} \mathrm{~d} \mu$, then

$$
\int P_{n}(x) P_{m}(x) \mathrm{d} \mu=0
$$

whenever $n \neq m$. These polynomials must satisfy a three-term recurrence relation of the form

$$
P_{n+1}(x)=\left(x-b_{n}\right) P_{n}(x)-\lambda_{n} P_{n-1}(x),
$$

with $P_{0}(x)=1$ and $P_{1}(x)=x-b_{0}$. Viennot showed $[14,15]$ that for any sequence $\left\{\mu_{n}\right\}$-which are called the moments —one can interpret the moment $\mu_{n}$ as the generating function for weighted Motzkin paths of length $n$ in which up steps have weight 1 , horizontal steps of height $k$ have weight $b_{k}$, and down steps of height $k$ have weight $\lambda_{k}$; then the polynomials in (1) will be orthogonal with respect to $\left\{\mu_{n}\right\}_{n \geq 0}$.

Many classical combinatorial sequences have been interpreted as the moment sequences for a set of orthogonal polynomials, and the corresponding orthogonality relation proved with a sign-reversing involution. In particular, it is known that:

- If $\mu_{2 n+1}=0$ and $\mu_{2 n}=C_{n}$, the Catalan number, then $b_{n}=0$ and $\lambda_{n}=1$; the corresponding polynomials are Chebyshev polynomials of the second kind [2], which may be defined by

$$
U_{n+1}(x)=x U_{n}(x)-U_{n-1}(x),
$$

with $U_{0}(x)=1$ and $U_{1}(x)=x$. These moments are $N C M_{0}(n)$.

- If $\mu_{2 n+1}=0$ and $\mu_{2 n}=(2 n-1)$ !!, then $b_{n}=0$ and $\lambda_{n}=n$; the corresponding polynomials are Hermite polynomials [14]. These moments are $N C M_{\infty}(n)$.

- If $\mu_{n}=M_{n}$, the $n$-th Motzkin number, then $b_{n}=1, \lambda_{n}=1$; the corresponding polynomials are shifted Chebyshev polynomials of the second kind: $U_{n}(x-1)$. See [3, section 4.1]. These moments are $N C P_{0}(n)$. 
- If $\mu_{n}=B_{n}$, the number of partitions of $[n]$, then $b_{n}=n+1$ and $\lambda_{n}=n$; the corresponding polynomials are Charlier polynomials (with $a=1$ ) [14]. These moments are $N C P_{\infty}(n)$.

With these observations, it is natural to try to use, say, $N C M_{k}(n)$ as a sequence of moments. Letting $k$ go from 0 to infinity would then allow us to interpolate between Chebyshev polynomials and Hermite polynomials; using $N C P_{k}(n)$ would give the corresponding interpolation between shifted Chebyshev and Charlier polynomials.

If we use $N C M_{2}(n)$ for the moments, then we have $b_{n}=0, \lambda_{2 n+1}=1$, and $\lambda_{2 n}=2$. This follows from the work of Kim and Zeng [6]: use $U_{n}(x, 2)$ in their paper. In their paper, they derive formulas for the moments of $U_{n}(x, 2)$ which are the same as known formulas for $\mathrm{NCM}_{2}(n)$, which are the little Schröder numbers.

If we attempt to do the same with $N C M_{3}(n)$, we get stuck: since $N C M_{3}(2 n+1)=0$, we know that $b_{n}=0$, but the $\lambda_{n}$ sequence starts with

$$
1,2, \frac{5}{2}, \frac{3}{10}, \frac{76}{5},-\frac{680}{57},-\frac{2311}{7752}, \frac{1246001}{314296}, \frac{114710016}{151553069}, \ldots
$$

Not only are some $\lambda_{n}$ 's fractions, but some are negative, which means prospects for polynomials with nice combinatorics are dim.

Let us try the same line of attack with $k$-distant noncrossing partitions. Using $N C P_{1}(n)$-Catalan numbers-for a set of moments, we get a shifted version of Chebyshev polynomials of the second kind: $b_{0}=1$, all other $b_{n}=2$, and all $\lambda_{n}=1$. These polynomials can be written $U_{n}(x-2)$, with slightly different initial conditions: $U_{0}(x)=1$ and $U_{1}(x)=x-1$. The easiest way to see why these recurrence coefficients and initial conditions are orthogonal with respect to the Catalan numbers is with a bijection between Motzkin paths of length $n$ with the above weighting and Dyck paths of length $2 n$ : take each up step $U$ and make it $U U$, take each down step $D$ and make it $D D$, and take each horizontal step $H$ and make it either $U D$ or $D U$ - except for the horizontal step at height zero, which can only be made into $U D$. This process turns a weighted Motzkin path of length $n$ into a Dyck path of length $2 n$ and is easily shown to be a bijection.

When using $N C P_{2}(n)$ and $N C P_{3}(n)$ as the moments, we again get some fractional coefficients, but they seem much nicer. We have computed the following with Maple: if $\mu_{n}=N C P_{2}(n)$ then

$$
\begin{aligned}
& \left\{b_{n}\right\}_{n \geq 0}=\left\{1,3-1,3-\frac{1}{2}, 3-\frac{1}{10}, 3-\frac{1}{65}, 3-\frac{1}{442}, 3-\frac{1}{3026}, \ldots\right\} \text { and } \\
& \left\{\lambda_{n}\right\}_{n \geq 1}=\left\{1,1+1,1+\frac{1}{4}, 1+\frac{1}{25}, 1+\frac{1}{169}, 1+\frac{1}{1156}, 1+\frac{1}{7921}, \ldots\right\}
\end{aligned}
$$

if $\mu_{n}=N C P_{3}(n)$ then

$$
\left\{b_{n}\right\}_{n \geq 0}=\{1,2,3,3,3, \ldots\} \quad \text { and } \quad\left\{\lambda_{n}\right\}_{n \geq 1}=\{1,2,2,2,2, \ldots\} .
$$

The first case is very interesting. The sequences of denominators of $b_{n}$ 's and $\lambda_{n}$ 's appear in A064170 and A081068 respectively. Based on the above evidence, we make the following conjectures.

Conjecture 6.1. If $\mu_{n}=N C P_{2}(n)$ then $b_{0}=1, b_{1}=2, \lambda_{1}=1$, and for $n \geq 2$

$$
b_{n}=3-\frac{1}{F_{2 n-1} F_{2 n-3}} \quad \text { and } \quad \lambda_{n}=1+\frac{1}{\left(F_{2 n-3}\right)^{2}} \text {, }
$$


where $F_{n}$ is the $n$-th Fibonacci number, i.e., $F_{n+1}=F_{n}+F_{n-1}$ and $F_{1}=F_{2}=1$.

Conjecture 6.2. If $\mu_{n}=N C P_{3}(n)$ then $b_{0}=1, b_{1}=2, b_{2}=3, \lambda_{1}=1, \lambda_{2}=2$, and, for $n \geq 3, b_{n}=3$ and $\lambda_{n}=2$.

\section{$7 \quad k$-distant $r$-crossing}

Chen et al. [1] considered a different kind of crossing number. Our definition of $k$-distant crossing can be applied to their definition.

Let $k \geq 0$ and $r \geq 2$ be integers. A $k$-distant $r$-crossing is a set of $r$ edges $\left(i_{1}, j_{1}\right),\left(i_{2}, j_{2}\right), \ldots,\left(i_{r}, j_{r}\right)$ such that $i_{1}<i_{2}<\cdots<i_{r} \leq j_{1}<j_{2}<\cdots<j_{r}$ and $j_{1}-i_{r} \geq k$. Similarly, a $k$-distant $r$-nesting is a set of $r$ edges $\left(i_{1}, j_{1}\right),\left(i_{2}, j_{2}\right), \ldots,\left(i_{r}, j_{r}\right)$ such that $i_{1}<i_{2}<\cdots<i_{r} \leq j_{r}<j_{r-1}<\cdots<j_{1}$ and $j_{r}-i_{r} \geq k$. In [1], they defined an $r$-crossing and an enhanced $r$-crossing, which are a 1-distant $r$-crossing and a 0 -distant $r$-crossing respectively.

Let $\operatorname{DCR}_{k}(\pi)$ (resp. $\operatorname{DNE}_{k}(\pi)$ ) be the maximal $r$ such that $\pi$ has a $k$-distant $r$-crossing (resp. $k$ distant $r$-nesting). Let $f_{n, S, T}(k ; i, j)$ denote the number of partitions $\pi$ of $[n]$ such that $\operatorname{DCR}_{k}(\pi)=i$, $\operatorname{DNE}_{k}(\pi)=j, \mathcal{O}(\pi)=S$ and $\mathcal{C}(\pi)=T$. Chen et al. [1] proved that $f_{n, S, T}(k ; i, j)=f_{n, S, T}(k ; j, i)$ for $k=0,1$. Krattenthaler [8] extended this result using growth diagrams.

Using Krattenthaler's growth diagram method, we can get the following theorem.

Theorem 7.1. Let $n \geq 1$ and $k \geq 0$ be integers. Then

$$
f_{n, S, T}(k ; i, j)=f_{n, S, T}(k ; j, i) .
$$

\section{References}

[1] William Y. C. Chen, Eva Y. P. Deng, Rosena R. X. Du, Richard P. Stanley, and Catherine H. Yan. Crossings and nestings of matchings and partitions. Trans. Amer. Math. Soc., 359(4):1555-1575 (electronic), 2007.

[2] Myriam de Sainte-Catherine and Gérard Viennot. Combinatorial interpretation of integrals of products of Hermite, Laguerre and Tchebycheff polynomials. In Orthogonal polynomials and applications (Bar-le-Duc, 1984), volume 1171 of Lecture Notes in Math., pages 120-128. Springer, Berlin, 1985.

[3] Dan Drake. Towards a combinatorial theory of multiple orthogonal polynomials. $\mathrm{PhD}$ thesis, University of Minnesota, August 2006.

[4] P. Flajolet. Combinatorial aspects of continued fractions. Discrete Math., 32(2):125-161, 1980.

[5] Anisse Kasraoui and Jiang Zeng. Distribution of crossings, nestings and alignments of two edges in matchings and partitions. Electron. J. Combin., 13(1):Research Paper 33, 12 pp.+, 2006.

[6] Dongsu Kim and Jiang Zeng. Combinatorics of generalized Tchebycheff polynomials. European J. Combin., 24(5):499-509, 2003.

[7] Jang Soo Kim. Bijections on 2-distant noncrossing partitions. preprint. 
[8] C. Krattenthaler. Growth diagrams, and increasing and decreasing chains in fillings of Ferrers shapes. Adv. in Appl. Math., 37(3):404-431, 2006.

[9] Toufik Mansour and Simone Severini. Enumeration of $(k, 2)$-noncrossing partitions. Discrete Mathematics, 308(20):4570-4577, October 2008.

[10] Jon McCammond. Noncrossing partitions in surprising locations. American Mathematical Monthly, 113(7):598-610, August 2006.

[11] Rodica Simion. Noncrossing partitions. Discrete Math., 217(1-3):367-409, 2000.

[12] N. J. A. Sloane. The on-line encyclopedia of integer sequences, 2008. Published electronically at www.research.att.com/ njas/sequences/.

[13] Richard P. Stanley. Enumerative combinatorics. Vol. 2, volume 62 of Cambridge Studies in Advanced Mathematics. Cambridge University Press, Cambridge, 1999. With a foreword by GianCarlo Rota and appendix 1 by Sergey Fomin.

[14] Gérard Viennot. Une théorie combinatoire des pôlynomes othogonaux generaux, Sep-Oct 1983. Notes from a conference given at the Université du Québec à Montréal.

[15] Gérard Viennot. A combinatorial theory for general orthogonal polynomials with extensions and applications. In Orthogonal polynomials and applications (Bar-le-Duc, 1984), volume 1171 of Lecture Notes in Math., pages 139-157, Berlin, 1985. Springer.

[16] Sherry H.F. Yan. Schröder paths and pattern avoiding partitions. arXiv:0805.2465, 2008. 